\title{
Weak Signal Detection with Duffing Oscillator Based on Virtual Instrument Technology
}

\author{
http://dx.doi.org/10.3991/ijoe.v11i1.4114 \\ Xuanchao Liu, Wenlong Ni \\ Xi'an Shiyou University, Xi'an, Shaanxi province, China
}

\begin{abstract}
To obtain new ways of detecting weak signals, we analyzed the motion of a Duffing oscillator in different input states by solving the Duffing equation and then elaborating on the basic principles of weak signal detection based on Duffing oscillator phase-change characteristics. The experiment that shows how to achieve weak signal detection with a Duffing oscillator based on virtual instrument technology and then discusses the impact on signal detection coming from Gaussian white noise and how to implement weak signal detection with noise. The results show that a Duffing oscillator not only can be effective for detection of weak signals in the background of strong noises, but it also has high performance to cost ratio to achieve it with virtual instrument technology. Compared to existing methods, it can greatly improve the detection results and has broad application.
\end{abstract}

Index Terms-weak signal detection, white noise, chaos, Duffing oscillator, virtual instrument.

\section{INTRODUCTION}

Weak signal detection is an important part of information technology and has widespread applications. "Weak" refers to not only small signal amplitude, but also that the small signal is overwhelmed by powerful background noise. To achieve detection of weak signals, human beings have done long-term research, on one hand analyzing the causes and distribution of noise and on the other hand researching characteristics of the measured signal to find a new approach that uses the differences to look for useful signals from background noise. Currently, the main weak signal detection methods are the following [1-4]:

\section{A. Narrowband filter detection}

If the signal spectrum is narrow and the distributed noise spectrum is wide, using narrow-band filters that can only pass narrow-band signals and filter out the noise outside the pass band significantly improved the signal to noise ratio to detect the useful signal buried in the noise. This method is easy to implement, but the instability of the filter center frequency has a greater impact in a narrow-band, so its application test results are subject to certain restrictions.

\section{B. Single-frequency lock detection}

Using the features of the single frequency or narrowband signal, conducting narrow-band processing first and then re-using cross-correlation principle finds a signal's cross-correlation processing. The frequency of the reference signal is the same as the detected signal, so the useful signal buried in the noise can be detected. This method is particularly suitable for single-frequency signal processing.

\section{Correlation detection method}

The feature of signals being related was used to achieve weak signal detection. This method is divided two ways: self-correlation detection and cross-correlation detection. Self-correlation detection uses an autocorrelation operation to remove noise based on the signal's periodic characteristics and the noise's random characteristics. Cross-correlation detection is based on the known frequency of the signal to be detected. Selecting a reference signal with the same frequency first and then making the cross-correlation operations between the reference signal and the signal to be detected can achieve the purpose of removing the noise. The results of a crosscorrelation detection method are better than selfcorrelation detection.

\section{Sampling integration method}

This method uses the feature that the signal is periodic and noise is random in a time domain. It divides periodic signals into time intervals and then samples the signals at these time intervals. It averages the sample values that are in the same position in each cycle using analog circuits or computer processing. This method can enhance useful signals and suppress noise to achieve effective detection for signals.

As information technology continues to evolve, people are exploring a variety of weak signal detection methods: detection methods based on wavelet transformation, detection based on fuzzy mathematics, artificial neural network-based detection, and in recent years, Duffing oscillator detection based on chaos theory has been considered and is achieving a lot of the results. Chaos detection method is a new signal processing method that is completely different from the existing variety of new signal detection methods. Research shows that the method has good detection results, is easier to achieve, and has broad application prospects. But the vast majority of research is still in the exploratory stage of theory and is less in relation to the physical realization $[5,6]$. This paper discusses the application of a Duffing oscillator in weak signal detection based on virtual instrument technology.

\section{WEAK SIGNAL DETECTION THEORY BASED ON DUFFING OSCILLATOR}

People have identified a chaotic phenomenon in the study of nonlinear dynamical systems. Behavior of chaotic dynamical systems is extremely sensitive for the initial parameters, and this feature is used in detection of 
weak signals [7,8]. A Duffing oscillator, because of its clear physical meaning and easy implementation, has become a hotspot of weak signal detection research.

A Duffing oscillator is a physical model of the soft spring oscillator with a non-linear restoring force. Oscillation occurs when a Duffing oscillator is excited by an external periodic signal. A Duffing equation is a mathematical model of weak damping, which describes the damping motion of a Duffing oscillator. The specific expression of the form is as follows:

$$
\chi^{\prime \prime}+\kappa \chi^{\prime}-\chi+\chi^{3}=\gamma \sin (t)
$$

Among which, $\gamma \cos (t)$ is the driving motivation for the cycle, $\mathrm{k}$ is the damping ratio, and $-\mathrm{x}(\mathrm{t})+\mathrm{x} 3(\mathrm{t})$ is the non-linear restoring force. We solved equation (1) by using MATLAB and then discovered that a Duffing oscillator has similar forms of differential equations compared to a general oscillator with linear restoring force; however, its features have undergone a fundamental change, and the most typical manifestation is the emergence of periodic motion and chaotic motion. In the case of a fixed $\mathrm{k}$ ( such as: $\mathrm{k}=0.5$ ), with $\gamma$ changing from small to large, the phase diagram of the system will be a homoclinic trajectory, a bifurcation trajectory, a chaotic trajectory, a critical cycle status, or changes in the large-scale periodic state. Especially when $\gamma$ changes to the critical period state $(\gamma=\gamma c)$, the system state will undergo a dramatic change. When $\gamma$ is less than $\gamma c$, the system is in a chaotic state, shown in Figure 1. When $\gamma$ is greater than $\gamma \mathrm{c}$, the system will enter the cycle of state, shown in Figure 2.

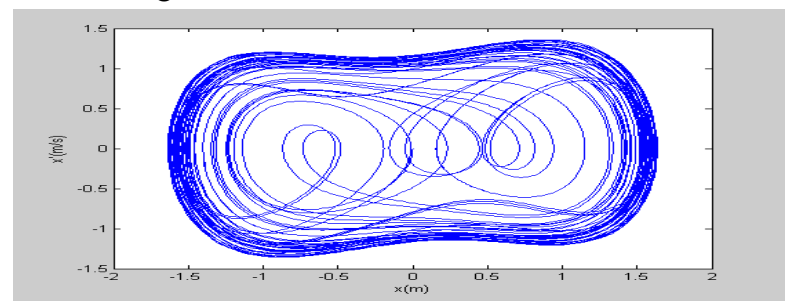

a. Phase orbit in a chaotic state

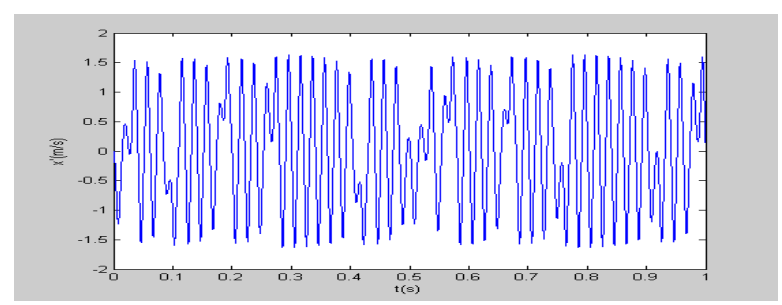

b. Time-domain output in a chaotic state

Figure 1 Phase orbit in a chaotic state

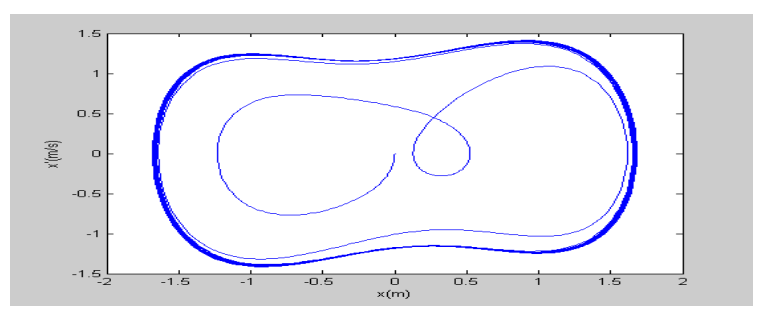

a. The great periodic state

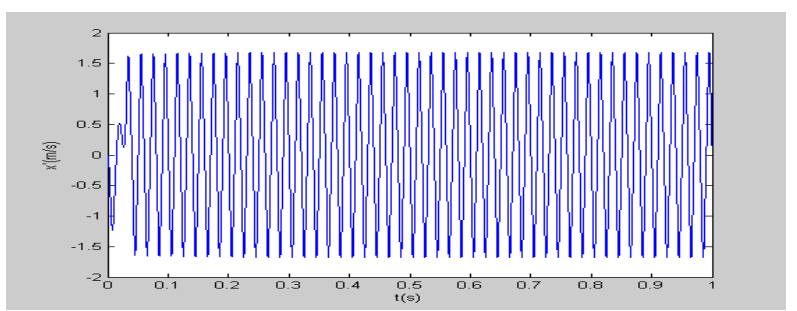

b. Time-domain output in great periodic state

Figure 2 Phase orbit in great periodic state

The process of these dramatic changes could be used for weak signal detection. Because if a reference signal with the same frequency and phase as the detected signal is added and the amplitude is equivalent or slightly smaller than the point $\gamma_{\mathrm{c}}$, when the weak signal being detected is injected in the Duffing oscillator, it may lead the system's state to change from chaotic status to a periodic state. When the system is in a periodic state, the phase trajectory can be landed on the largest-scale track, and its behavior is the period signal in a time domain, which is very different from the chaotic state. By distinguishing between the two different states, we can achieve weak signal detection.

\section{WEAK SIGNAL DETECTION EXPERIMENT ON VIRTUAL INSTRUMENT}

Virtual instrument technology, which can be realized by LabVIEW language, is an effective means for realizing weak signal detection with a Duffing oscillator [9,10]. Figure 3 is a virtual instrument experiment platform of weak signal detection by LabVIEW language.

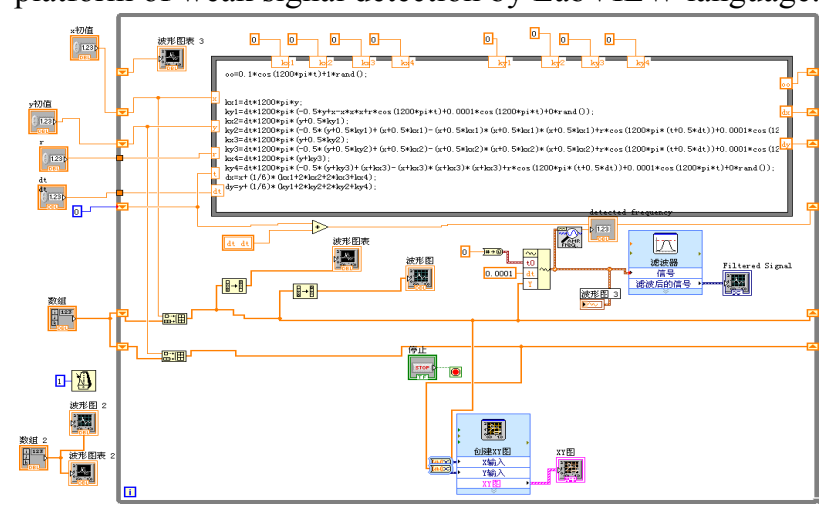

Figure 3 Duffing oscillator experiment platform by LabVIEW

\section{A. Activeness analysis of Duffing oscillator detection in an ideal situation}

The so-called ideal situation is that the reference signal has the same frequency and phase as the signal being detected with no noises. We can investigate the signal detection effects through solving a Duffing equation using LabVIEW software.

The research shows that $\gamma$ is taken after the decimal point three, four, and six significant figures: obtained $\gamma \mathrm{c}$ is $0.787 \mathrm{~V}, 0.7868 \mathrm{~V}, 0.786786 \mathrm{~V}$ when the Duffing equation is solved dynamically. Through $\gamma \mathrm{c}$ we can accurately determine the chaotic state and large cycle status, but the relationship between resolution and detection time is contradictory; namely, the addition of significant figures in the number can increase signal detection accuracy, but when $\gamma>\gamma \mathrm{c}$, a Duffing oscillator 
PAPER

enters the big cycle state slowly, which greatly reduces the signal detection efficiency. A better approach is to give a compromise. So when $\gamma \mathrm{c}$ is four significant figures $(0.7868 \mathrm{~V})$, the Duffing oscillator state is shown in Figure 4 , but when the amplitude of the tested signal is to increase $0.0001 \mathrm{~V}$, the Duffing oscillator state changes quickly into what is shown in Figure 5. These show that the signal can be detected effectively; its detection sensitivity $\Delta V$ is $0.0001 \mathrm{~V}$.

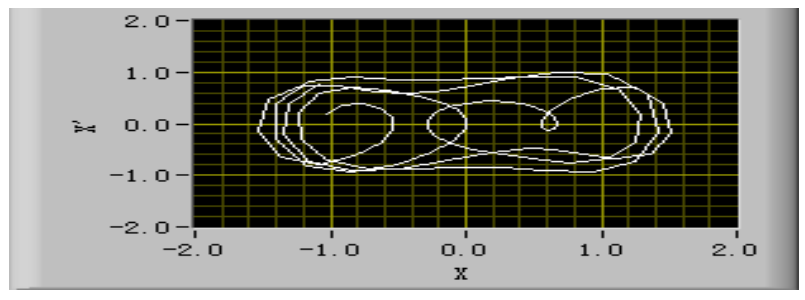

a. Phase orbit in chaotic state

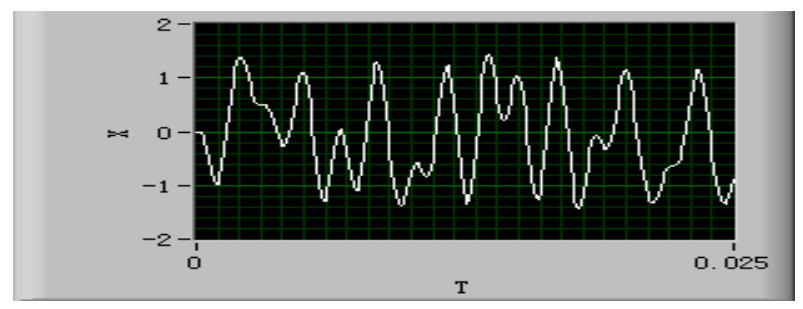

b. Time-domain output in chaotic state

Figure 4 Phase orbit in chaotic state

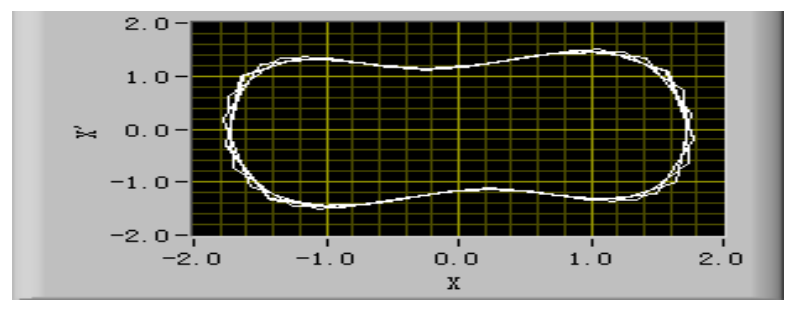

a. The great periodic state

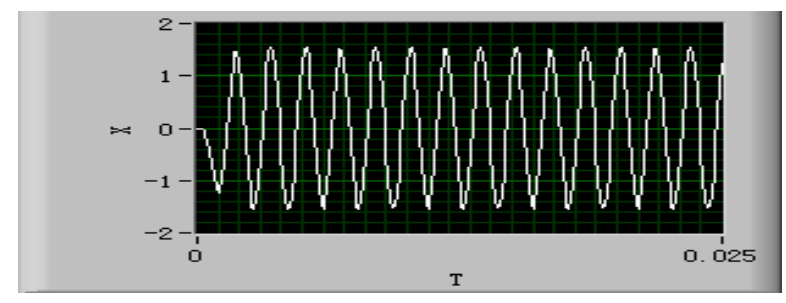

b. Time-domain output in great periodic state

Figure 5 Phase orbit in great periodic state

\section{B. The impact of noise on signal detection}

Taking into account the efficiency and the accuracy of the signal detection, we also used the four digits after the decimal point for $\gamma$ to test. As mentioned before, when $\gamma \mathrm{c}$ $=0.7868 \mathrm{~V}$, the Duffing oscillator enters the critical chaotic state, when the signal amplitude is $0.0001 \mathrm{~V}$ and the noises being added are about $30 \mathrm{db}$, as shown in Figure 6. The Duffing oscillator state is shown in Figure 7 , indicating that the state can enter the big cycle when adding $30 \mathrm{db}$ of Gaussian white noise to the detection signal. When adding more than $30 \mathrm{db}$ Gaussian white noise to the detection signal, the Duffing oscillator goes back to the chaotic state. These show that Duffing oscillator detection not only can detect weak signals, but it also has very strong noise suppression.

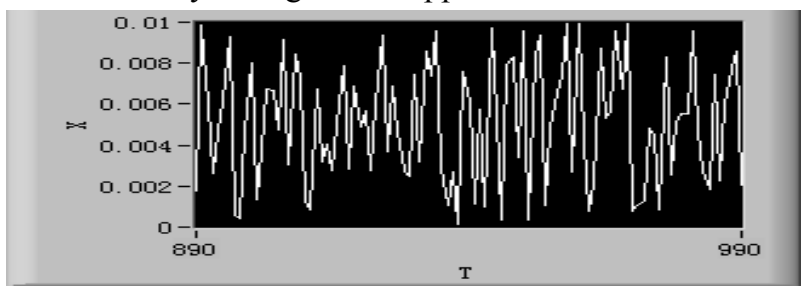

Figure 6 Signal with Gaussian white noises

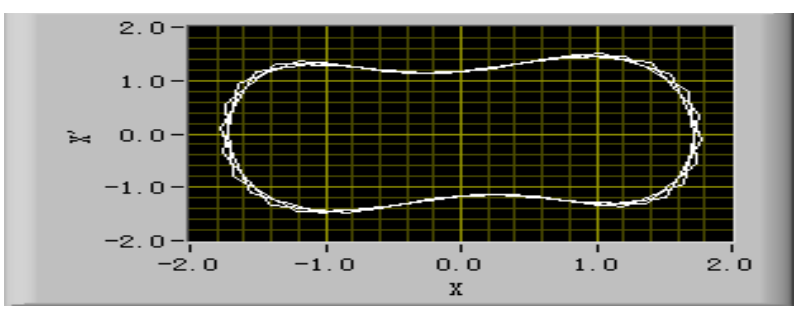

Figure 7 The output with great periodic state

C. Duffing oscillator characteristics in case of detection signal and reference signal existing frequency difference

We discussed the basic principle of weak signal detection based on the Duffing oscillator under the ideal situation; namely, the frequency of the reference signal and the signal being detected are the same. Research on the Duffing oscillator indicates that the system will generate an intermittent chaos phenomenon if there is an extremely small frequency difference between the signal being detected and the reference signal, as shown in Figure 8.

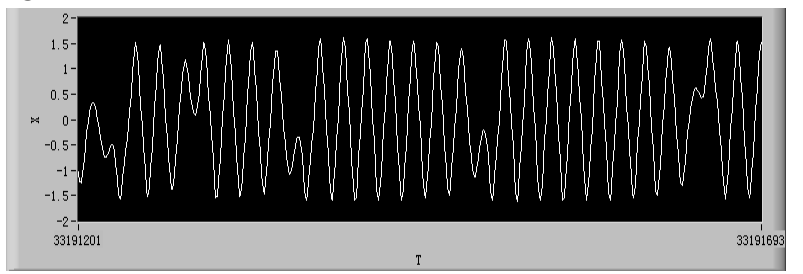

Figure 8 Intermittent chaos phenomenon of the chaotic status and the periodic status

The intermittent chaos phenomenon is that the chaotic state and the large-scale periodic state appeared alternately in the time domain. Essentially, the sum of the reference signal and the signal being detected produced a synthetic signal; the amplitude of the synthetic signal grows greater than and declines less than the marginal value $\gamma_{c}$ alternately in time. According to the following Duffing equation, we can analyze the principle of the intermittent chaos phenomenon clearly.

Equation (1) is rewritten as follows:

$$
\chi^{\prime \prime}+\kappa \chi^{\prime}-\chi+\chi^{3}=F(t)=F_{r}(t)+F_{i}(t)
$$

$$
\begin{gathered}
F(t)=\gamma_{r} \sin (t)+\gamma_{i} \sin ((1+\Delta \omega) t+\varphi) \\
=\gamma(t) \sin (t+\lambda(t))
\end{gathered}
$$


Among which $\Delta \omega=\omega_{i}-\omega_{r}, \gamma_{r}$ is the amplitude of the cycle reference driving signal (equal to or slightly smaller than the marginal value $\gamma_{c}$ ), $\gamma_{i} \sin ((1+\Delta \omega) t+\varphi)$ represents the external signal being detected, $\gamma_{i}$ is its amplitude, and $\varphi_{\text {is its initial }}$ phase.

$$
\begin{gathered}
\gamma(t)=\sqrt{\gamma_{r}^{2}+2 \gamma_{r} \gamma_{i} \cos (\Delta \omega \cdot t+\varphi)+\gamma_{i}^{2}} \\
\gamma_{r}-\gamma_{i} \leq \gamma(t) \leq \gamma_{r}+\gamma_{i} \\
\lambda(t)=\operatorname{arctg} \frac{\gamma_{i} \sin (\Delta \omega \cdot t+\varphi)}{\gamma_{r}+\gamma_{i} \cos (\Delta \omega \cdot t+\varphi)} \\
\gamma_{i<<} \gamma_{r}
\end{gathered}
$$

Although there is a variable in formula (5), because of $\gamma_{i<<} \gamma_{r}, \lambda(t)$ is very small, and its changes have a small influence on the system, which could be ignored, so:

$$
F(t) \approx \gamma(t) \sin (t)
$$

In the case of a small frequency difference between the signal being detected and the reference signal, according to the formula (4), if $\gamma_{c}$ as the following: $\gamma_{r}-\gamma_{i} \leq \gamma_{c} \leq \gamma_{r}+\gamma_{i}$, we can see that the system state can come into the large-scale periodic state when $\gamma(t)$ is greater than chaotic threshold $\gamma_{c}$, and sometimes the system state will come into chaotic status when $\gamma(t)$ is less than chaotic threshold $\gamma_{c}$; therefore, the system state appears as a specific intermittent chaos phenomenon. The large-scale periodic state and chaotic state will change in cyclical way; the cycle $\mathrm{T}$ is shown as formula (7).

$$
\mathrm{T}=2 \pi / \Delta \omega
$$

When $\Delta \omega$ is larger, the speed of the changing state is quick. The system has difficulty holding the large-scale periodic state for a long time. So it is hard to identify the phenomenon of the intermittent chaos. If increasing $\Delta \omega$ further, the system state can come into a continuous chaotic state, just like responding to some noises. But when $\Delta \omega$ is very small, such as $\Delta \omega / \omega<0.001$, the $\gamma(t)$ changes very slowly. So the system has a good response to the signal being detected.
When $\Delta \omega$ is small, the intermittent chaos phenomenon is easy to identify. Not only can we determine whether a signal being detected exists, but we can also get frequency differences between the signal being detected and the reference driving signal by formula (7). According to the intermittent chaos cycle $\mathrm{T}$, we also can achieve measurement of the weak signal's frequency.

The result shows that in the cases in which we cannot guarantee the same frequency between the signals being detected and the reference signals, we can just use this feature to detect the signal. It is easily realized with virtual instrument technology, but the detection efficiency is lower.

\section{EXPERIMENTAL RESULTS AND DISCUSSION}

The simulation results revealed the Duffing oscillator's operating characteristics and verified the validity of the weak signal detection. In the ideal case, using MATLAB to solve a Duffing equation will identify Duffing oscillator output behavior in the case of input signal amplitude changing from small to big, especially through virtual instrument technology to achieve the Duffing oscillator state dynamically. In the chaotic state, the input signal varies widely and Duffing oscillator is still in a chaotic state, but there is always a signal amplitude of a point $\gamma \mathrm{c}$. As the input signal changes are small at this point, it will cause a sudden phase transition of the Duffing oscillator. When $\gamma>\gamma \mathrm{c}$, the Duffing oscillator will change suddenly from chaos to a large cycle state, and when $\gamma<\gamma \mathrm{c}$, it will suddenly change from a great cycle state to a chaotic state. And here, adding a lot of noise does not transform the phase of the Duffing oscillator, which indicates where it is sensitive for signals and is a great suppression for noise. According to this feature, first select the reference signal with an amplitude less than $\gamma \mathrm{c}$, making the detection signal and reference signal superposed, and then when the input signal and reference signal are inputted with the same frequency, a small increase of input signal will cause phase transitions to achieve weak signal detection. In the experiment, entering the signal with a $-30 \mathrm{db}$ signal to noise ratio caused a phase transition, which fully illustrates that it has a unique sensitivity for single-frequency sinusoidal signals buried in noise and suppression capabilities for random noise. When a frequency difference between the input signal and the reference signal exists, there will be intermittent chaos. Taking advantage of this phenomenon not only can detect weak signals, but it can also accurately detect the frequency of the signal according to an intermittent cycle. When the system is in the great cycle state, output signals in the time domain pass through the zero point with the same interval, and when the system is in a chaotic state, the output signal zerocrossing time interval is variable, so we can determine that the system is in a large-scale periodic state or in a chaotic state by comparing the output signal zerocrossing time intervals. But how to ensure that the reference signal has the same or similar frequency and phase with the input signal still needs to be studied.

\section{CONCLUSION}

This paper analyzed the operating characteristics of the Duffing oscillator, identifying the basic principles of detecting weak signals from strong background noise and 
also examining the signal detection method when intermittent chaos occurs in the case of existing frequency differences between the signal being detected and the reference signal. The results show that the Duffing oscillator's state from chaotic to great cycle is sensitive to the changes of signal amplitude, but it is insensitive to noise. We can take full advantage of this feature to conduct weak signal detection. Detection sensitivity of this method is high compared to the commonly used signal detection methods, but it also has high performance to cost ratio to achieve it with virtual instrument technology.

\section{REFERENCES}

[1] Jinzhan Gao. Weak Signal Detection. Tsinghua University Press, 2004.

[2] Guanyu Wang, Dajun Chen, Jianya Lin, Xing Chen. "The application of chaotic oscillators to weak signal detection", IEEE Trans. Ind. Elect., vol. 46, no. 2, pp. 440-444, 1999. http://dx.doi.org/10.1109/41.753783

[3] Guanyu Wang, Wei Zheng, Sailing He. "Estimation of amplitude and phase of a weak signal by using the property of sensitive dependence of initial conditions of a nonlinear oscillator". Signal Proc., vol. 82, no. 1, pp. 103-115, 2002. http://dx.doi.org/10.1016/ S0165-1684(01)00166-9

[4] Tao Xie, Xueye Wei. "Study on reliability of chaotic oscillator in weak signal detection". Chinese J. Sci. Instrum., vol. 29, no. 6, pp. 1265-1269, 2008.

[5] Wenli Zhao, Zhenqiang Huang, Jingxiao Zhao. "Electronic circuit realization of weak signal detection based on Duffing oscillator". $J$. Circ. Syst., vol. 16, no. 6, pp. 120-124, 2011.
[6] Yongsheng Wang, Zicai, Sunjin Xiao, Hongda Fan. "Circuit simulation of duffing cha otic system", Circ. Syst., vol. 13, no. 1, pp. 132-135, 2008.

[7] Donald L. "Birx. Chaotic oscillators and CMFFNS to signal detection in noise environments", IEEE Int. Joint Conf. Neural Networks, 1992, vol. 2, pp. 881-888.

[8] Li Liu, Jun Sun. "Weak signal detection method study based on Chaotic oscillator". J. Shenyang Agr. Univ., vol. 36, no. 6, pp. 667-668, 2005.

[9] Jian Fan, Wenli Zhao, Wanqiang Wang. "Study on the weak simusoidal signal detection property using duffing chaos system" Acta Phys. Sin., vol. 62. no. 18, 2013, 180502.1-180502.6.

[10] Chunyan Nie, Yaowu Shi. "Design of chaos simulation instrument based on virtual instrument". Elect. Meas. \& Instrum., vol. 42, no. 469, pp. 35-37, 2005.

\section{AUTHORS}

Xuanchao Liu is with the Electronic Engineering Institute of Xi'an Shiyou University, Xi'an, Shaanxi province, China (e-mail: 13186172127@163.com).

Wenlong Ni is with the Key Laboratory of Downhole Drilling Engineering of CNPC, Xi'an Shiyou University, Xi'an, Shaanxi province, China (e-mail: taxuelangzi110@gmail.com).

This work is supported by the National Natural Science Foundation of China (50234030) and the Xi'an Sitan Petroleum Instruments Co. Submitted 14 August 2014. Published as resubmitted by the authors 25 January 2015. 